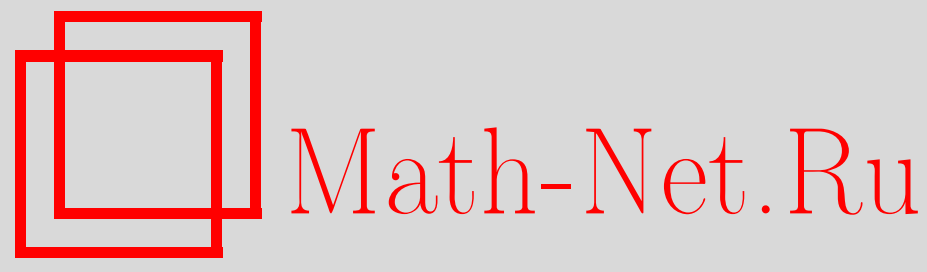

M. А. Лифшиц, Zh. Shi, Нижние функции эмпирического процесса и броуновского листа, Теория вероятн. и ее примен., 2003, том 48, выпуск 2, 321-339 DOI: https://doi.org/10.4213/tvp287

Использование Общероссийского математического портала MathNet.Ru подразумевает, что вы прочитали и согласны с пользовательским соглашением

http: //www . mathnet.ru/rus/agreement

Параметры загрузки:

IP : 54.89 .56 .158

26 апреля 2023 г., 09:49:19

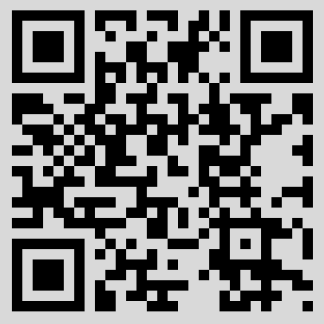




\title{
НИЖНИЕ ФУНКЦИИ ЭМПИРИЧЕСКОГО ПРОЦЕССА И БРОУНОВСКОГО ЛИСТА ${ }^{1)}$
}

\begin{abstract}
В терминах интегральных критериев дана полная характеризация классов нижних функций двупараметрического броуновского листа (поля Винера-Ченцова) и равномерного эмпирического процесса. Результат, относящийся к броуновскому листу, можно рассматривать как решение весьма специального случая следующей общей задачи: найти скорость ухода на бесконечность произвольного бесконечномерного броуновского движения (в общем случае этот вопрос остается открытым). Наш результат для эмпирического процесса опровергает одну гипотезу из книги Шорака и Веллнера [18] и дает ответ на поставленный в ней вопрос.
\end{abstract}

Ключевые слова и фразы: скорость ухода на бесконечность, эмпирический процесс, бесконечномерное броуновское движение, броуновский лист, нижняя функция.

\section{1. Введение и основные результаты.}

1.1. Скорость ухода на бесконечность броуновских движений. Мы посвящаем эту статью столетию со дня рождения великого математика А. Н. Колмогорова. Интерес Андрея Николаевича к интегральным тестам для проверки асимптотических свойств случайных процессов хорошо известен - достаточно вспомнить знаменитый тест Колмогорова-Петровского-Эрдёша-Феллера для верхних функций винеровского процесса и случайных блужданий, см. [1]. Наша работа в терминах интегральных тестов решает некоторые давно стоявшие задачи, связанные со скоростью ухода на бесконечность броуновского листа и эмпирического процесса.

${ }^{*}$ С.-Петербургский государственный университет, механико-математический факультет, Библиотечная пл., 2, 198504 Старый Петергоф, Россия; Université Lille I, Statistique et Probabilités, Bât. M2, F-59655 Villeneuve d'ascq, France; e-mail: lifts@mail.rcom.ru

** Laboratoire de Probabilités UMR 7599, Université Paris VI, 4 place Jussieu, F-75252 Paris Cedex 05, France; e-mail: zhan@proba.jussieu.fr

1) Работа первого автора частично выполнена при финансовой поддержке грантов программы «Университеты России» (проект 99-26-11) и Российского фонда фундаментальных исследований (проекты 00-15-96019 и 02-01-00265). 
Рассмотрим стандартное броуновское движение $B_{d}:=\left\{B_{d}(t), t \geqslant 0\right\}$ со значениями в $\mathbf{R}^{d}$. Хорошо известно, что $B_{d}$ почти наверное невозвратно, т.е. $\left\|B_{d}(t)\right\| \rightarrow \infty$ при $t \rightarrow \infty$, где $\|\cdot\|$ обозначает евклидову норму, в том и только том случае, когда $d \geqslant 3$. Если последнее условие выполнено, то скорость ухода $B_{d}$ на бесконечность была определена Дворецким и Эрдёшем [10]. В дальнейшем, если не оговорено иное, «б.ч.» означает «бесконечно часто» при стремлении соответствующего параметра к бесконечности.

Теорема А (Дворецкий и Эрдёш [10]). Пусть $d \geqslant 3$. Тогда для любой неубывающей функиии $f>0$ верно

$$
\mathbf{P}\left\{\left\|B_{d}(t)\right\| \leqslant \frac{t^{1 / 2}}{f(t)} \quad \text { б.ч. }\right\}=\left\{\begin{array} { l } 
{ 0 } \\
{ 1 }
\end{array} \int ^ { \infty } \frac { d t } { t f ^ { d - 2 } ( t ) } \left\{\begin{array}{l}
<\infty \\
=\infty
\end{array} .\right.\right.
$$

Что происходит в пространствах бесконечной размерности? Пусть $X:=\{X(t), t \geqslant 0\}-$ броуновское движение в вещественном сепарабельном бесконечномерном пространстве $(E,\|\cdot\|)$ с $X(0)=0$. Иными словами, $X$ имеет стационарные независимые приращения, непрерывные траектории и нулевое среднее (т.е. для любого линейного непрерывного функционала $f$ и любого $t \geqslant 0$ верно $\mathbf{E}[f(X(t))]=0)$.

Следуюший бесконечномерный результат принадлежит Эриксону [12].

Теорема В (Эриксон [12]). Пусть $X-$ существенно бесконечномерное броуновское движение в $E$ u $X(0)=0$. Если $f$ - медленно меняюшаяся на бесконечности положительная непрерьвная функиия на $\mathbf{R}_{+}$, то для любого $\delta>0$ верно

$$
\begin{gathered}
\int^{\infty} \frac{\mathbf{P}\{\|X(1)\| \leqslant(1-\delta) / f(t)\}}{t f^{2}(t)} d t=\infty \Longrightarrow \mathbf{P}\left\{\|X(t)\| \leqslant \frac{t^{1 / 2}}{f(t)} \text { б.ч. }\right\}=1, \\
\int^{\infty} \frac{\mathbf{P}\{\|X(1)\| \leqslant(1+\delta) / f(t)\}}{t f^{2}(t)} d t<\infty \Longrightarrow \mathbf{P}\left\{\|X(t)\| \leqslant \frac{t^{1 / 2}}{f(t)} \text { б. ч. }\right\}=0 .
\end{gathered}
$$

Эта теорема полезна для определения функции $f$, задающей правильный порядок ухода $X$, в случае, когда мы располагаем достаточно хорошей информацией о поведении $\mathbf{P}\{\|X(1)\| \leqslant \varepsilon\}$ при $\varepsilon \rightarrow 0+$. (См. работу Кокса [8] о существовании таких функций.) Отметим, что оригинальный результат Эриксона является более общим - его можно применить к любой непрерывной полунорме на $E$, имеющей ранг не менее 3 по отношению к $X$.

Представляется очень интересной задачей найти необходимое $u$ достаточное условие, как это сделано в теореме А. Другими словами, что происходит в критическом случае $(\delta=0)$ ? До сих пор такие результаты не были известны. 
В этой статье мы изучаем один конкретный, но очень важный пример бесконечномерного броуновского движения. Рассмотрим броуновский лист (двумерное поле Винера-Ченцова) $\mathbf{M}:=\{\mathbf{M}(s, t), s \in[0,1]$, $t \geqslant 0\}$. Иначе говоря, $\mathbf{M}$ это центрированное гауссовское случайное поле с ковариацией

$$
\mathbf{E}\left[\mathbf{M}(s, t) \mathbf{M}\left(s^{\prime}, t^{\prime}\right)\right]=\min \left(s, s^{\prime}\right) \min \left(t, t^{\prime}\right) .
$$

Как известно, случайные поля можно интерпретировать как бесконечномерные процессы (см., например, [13]). Для каждого $t$ будем рассматривать

$$
X(t):=(s \mapsto \mathbf{M}(s, t), s \in[0,1])
$$

как элемент $C([0,1], \mathbf{R})$, пространства всех непрерывных функций на $[0,1]$ с равномерной нормой

$$
\|f\|:=\sup _{s \in[0,1]}|f(s)| .
$$

Важность именно этого броуновского движения объясняется его тесной связью (через процесс Кифера и КМТ-аппроксимацию) с эмпирическими процессами. Подробнее об этом будет сказано в разделе 4.

Поскольку $X(1)$ является стандартным броуновским движением на временном интервале $[0,1]$, классический результат Чжуна [7] дает

$$
\mathbf{P}\{\|X(1)\| \leqslant \varepsilon\} \sim \frac{4}{\pi} \exp \left(-\frac{\pi^{2}}{8 \varepsilon^{2}}\right), \quad \varepsilon \rightarrow 0,
$$

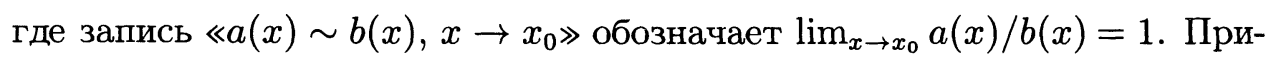
меняя теорему В к $\mathbf{M}$, находим, что для любой медленно меняющейся на бесконечности положительной непрерывной функции $f$ и для любого $\delta>0$ верно

$$
\begin{aligned}
& \int^{\infty} \frac{1}{t f^{2}(t)} \exp \left(-\frac{\pi^{2}+\delta}{8} f^{2}(t)\right) d t=\infty \Longrightarrow \mathbf{P}\left\{\|X(t)\| \leqslant \frac{t^{1 / 2}}{f(t)} \text { б.ч. }\right\}=1, \\
& \int^{\infty} \frac{1}{t f^{2}(t)} \exp \left(-\frac{\pi^{2}-\delta}{8} f^{2}(t)\right) d t<\infty \Longrightarrow \mathbf{P}\left\{\|X(t)\| \leqslant \frac{t^{1 / 2}}{f(t)} \text { б.ч. }\right\}=0 .
\end{aligned}
$$

Мы предлагаем следующий интегральный тест для случая $\delta=0$.

Теорема 1.1. Пусть $X$ определено в (1.1). Если функиия $f>0$ не убывает, то

$$
\int^{\infty} \frac{f^{6}(t)}{t} \exp \left(-\frac{\pi^{2}}{8} f^{2}(t)\right) d t\left\{\begin{array}{c}
<\infty \\
=\infty
\end{array} \Longleftrightarrow \mathbf{P}\left\{\|X(t)\| \leqslant \frac{t^{1 / 2}}{f(t)} \text { б. } .\right\}=\left\{\begin{array}{l}
0 \\
1
\end{array},\right.\right.
$$

где норма $\|\cdot\|$ определена в (1.2).

Присутствие в тесте необычной степени «6» указывает на достаточно экзотические свойства ухода броуновского листа на бесконечность. 
1.2. Скорость ухода на бесконечность эмпирического процесса. Объясним первоначальную мотивацию нашей работы. Ее исходной точкой была гипотеза Шорака и Веллнера [18] о нижних функциях эмпирических процессов. Пусть $\left\{\alpha_{n}(s), s \in[0,1]\right\}-$ равномерный эмпирический процесс (см. определение (4.1) в разделе 4). Нас интересует $\left\|\alpha_{n}\right\|:=\sup _{s \in[0,1]}\left|\alpha_{n}(s)\right|$, равномерная норма $\alpha_{n}$. Известно (см. работы А. А. Могульского [3] и Дж. Кэлбса [17]), что

$$
\liminf _{n \rightarrow \infty} \sqrt{\ln \ln n}\left\|\alpha_{n}\right\|=\frac{\pi}{\sqrt{8}} \quad \text { п.н. }
$$

Как обстоит дело с тестами для нижних функций $\left\|\alpha_{n}\right\|$ ? В книге Шорака и Веллнера [18], с. 530, высказана следующая гипотеза: для любой положительной неубывающей последовательности $\left(\lambda_{n}\right)$

$$
\mathbf{P}\left\{\left\|\alpha_{n}\right\| \leqslant \frac{1}{\lambda_{n}} \text { б.ч. }\right\}=\left\{\begin{array} { l } 
{ 0 } \\
{ 1 }
\end{array} \sum _ { n } \frac { \lambda _ { n } ^ { 3 } } { n } \operatorname { e x p } ( - \frac { \pi ^ { 2 } } { 8 } \lambda _ { n } ^ { 2 } ) \left\{\begin{array}{l}
<\infty \\
=\infty
\end{array} .\right.\right.
$$

Используя технику доказательства теоремы 1.1 , мы опровергаем гипотезу (1.5). В разделе 4 будет доказано, что правильный ответ таков:

Теорема 1.2. Пусть $\left(\lambda_{n}\right)$ не убывает. Тогда

$$
\mathbf{P}\left\{\left\|\alpha_{n}\right\| \leqslant \frac{1}{\lambda_{n}} \text { б.ч. }\right\}=\left\{\begin{array} { l } 
{ 0 } \\
{ 1 }
\end{array} \Longleftrightarrow \sum _ { n } \frac { \lambda _ { n } ^ { 7 } } { n } \operatorname { e x p } ( - \frac { \pi ^ { 2 } } { 8 } \lambda _ { n } ^ { 2 } ) \left\{\begin{array}{l}
<\infty \\
=\infty
\end{array} .\right.\right.
$$

Очевидно, эта теорема содержит (1.4) как частный случай.

1.3. Критические подпоследовательности. Скажем несколько слов об одном ключевом техническом аспекте работы. Согласно терминологии Бреймана [6], интегральный тест, с которым мы работаем в теоремах 1.1 и 1.2 , относится к категории «тонких》 в том смысле, что умножение $f$ на константу может изменить результат применения теста, в то время как тест Дворецкого-Эрдёша в теореме А не столь чувствителен. Обычно считается (см. однако замечания в разделе 5), что для тонкого интегрального теста достаточно следить за поведением процесса вдоль подпоследовательности моментов времени

$$
t_{i}:=\exp \left(\frac{i}{\ln i}\right)
$$

(или другой подобной подпоследовательности, для которой $\ln t_{i}$ сравним с $i / \ln i$ при больших $i$ ), которую можно назвать подпоследовательностью Эрдёша, отдавая должное его работе [11], посвященной доказательству колмогоровского теста. В частности, анализ подпоследовательности Эрдёша ведет к гипотезе (1.5). Разумеется, иногда используют такие варианты последовательности Эрдёша, как $\exp (\exp (i / \ln i)),-$ если процесс имеет временную структуру логарифмического типа. 
Мы показываем, что анализ подпоследовательности Эрдёша в нашем случае не работает. Как ни странно, в доказательстве теоремы 1.1 приходится использовать необычную подпоследовательность

$$
t_{i}:=\exp \left(\frac{i}{(\ln i)^{3}}\right) .
$$

Хотя тщательный анализ некоторых аспектов двумерного броуновского движения показывает, что последовательность (1.6) дает ключ к решению, у нас нет убедительного объяснения, почему a priori это должно быть так и почему в данном случае не работает анализ подпоследовательности Эрдёша. Безусловно, важную роль здесь играет бесконечномерная природа задачи, и нам представляется возможным, что подпоследовательность Эрдёша не будет работать ни для какой существенно бесконечномерной ситуации (см. дальнейшее обсуждение в разделе 5 ).

Материал статьи расположен следующим образом. Мы доказываем теорему 1.1 в разделе 2, принимая сначала на веру ключевую вероятностную оценку (предложение 2.1), в которой идет речь о вероятности того, что двумерное броуновское движение долгое время находится в узком параллелограмме. Доказательство предложения 2.1 составляет содержание раздела 3. Раздел 4 посвящен изучению нижних функций эмпирического процесса. В частности, здесь мы доказываем теорему 1.2 . Наконец, дополнительные замечания и вопросы собраны в разделе 5 .

В тексте статьи буква $c$ с различными индексами обозначает несущественные для наших целей положительные конечные постоянные. Мы также используем обычную запись $a_{i} \asymp b_{i}$ для обозначения соотношений $0<\liminf _{i} a_{i} / b_{i} \leqslant \lim \sup _{i} a_{i} / b_{i}<\infty$.

2. Доказательство теоремы 1.1. В этом разделе $\mathbf{M}=\{\mathbf{M}(s, t)$, $\left.s \in \mathbf{R}_{+}, t \in \mathbf{R}_{+}\right\}$обозначает броуновский лист, а $f>0$ считается неубывающей функцией, заданной на $\mathbf{R}_{+}$. Две части теоремы 1.1 состоят в следующем:

$$
\begin{gathered}
\int^{\infty} \frac{f^{6}(t)}{t} \exp \left(-\frac{\pi^{2}}{8} f^{2}(t)\right) d t<\infty \\
\Longrightarrow \mathbf{P}\left\{\|\mathbf{M}(\cdot, t)\| \leqslant \frac{t^{1 / 2}}{f(t)} \text { б.ч. }\right\}=0, \\
\int^{\infty} \frac{f^{6}(t)}{t} \exp \left(-\frac{\pi^{2}}{8} f^{2}(t)\right) d t=\infty \\
\Longrightarrow \mathbf{P}\left\{\|\mathbf{M}(\cdot, t)\| \leqslant \frac{t^{1 / 2}}{f(t)} \text { б.ч. }\right\}=1,
\end{gathered}
$$

где $\|\cdot\|$ обозначает, как и ранее, равномерную норму.

Доказательство импликации (2.1), которое основано на минимальном неравенстве типа применявшихся А. А. Могульским, требует лишь 
комбинирования достаточно стандартных приемов. Напротив, доказательство (2.2) является основным содержанием данной статьи. Работая с бесконечномерным броуновским движением, можно, конечно, использовать такие универсальные приемы работы с гауссовскими мерами, как неравенство Андерсона, но даже в частном случае броуновского листа из них не выводится (2.2). Мы будем использовать некоторые специальные свойства броуновского листа, связывающие его с $\mathbf{R}^{2}$-значным броуновским движением, параметризованным элементами $\mathbf{R}_{+}$.

Хорошо известно, что для интегральных тестов типа (2.1)-(2.2), не ограничивая общности, можно рассматривать только «критические функции». Поэтому в дальнейшем мы предполагаем, что

$$
\frac{\sqrt{\ln \ln t}}{2} \leqslant f(t) \leqslant \sqrt{\ln \ln t} \text { для } t \geqslant t_{0} .
$$

Строгое обоснование можно найти в [11] или [9].

Определим последовательность $\left(t_{i}\right)_{i \geqslant 2}$ соотношением

$$
t_{i}=\exp \left(\frac{i}{(\ln i)^{3}}\right)
$$

(Напомним, что это та самая последовательность, которая уже упоминалась в (1.6).) Легко подсчитать, что

$$
\frac{t_{i+1}}{t_{i}}-1 \asymp \frac{1}{(\ln i)^{3}} \asymp \frac{1}{f^{6}\left(t_{i}\right)}
$$

и что

$$
\int^{\infty} \frac{f^{6}(t)}{t} \exp \left(-\frac{\pi^{2}}{8} f^{2}(t)\right) d t<\infty \Longleftrightarrow \sum_{i} \exp \left(-\frac{\pi^{2}}{8} f^{2}\left(t_{i}\right)\right)<\infty .
$$

Теперь по очереди докажем обе половины, (2.1) и (2.2), теоремы 1.1.

Док аз а т ель с т в о соо тношен и я (2.1). Пусть функция $f$ такова, что $\int^{\infty}\left(f^{6}(t) / t\right) \exp \left(-\left(\pi^{2} / 8\right) f^{2}(t)\right) d t<\infty$. Пусть

$$
\sigma_{i}:=\inf \left\{t \geqslant t_{i}:\|\mathbf{M}(\cdot, t)\| \leqslant \frac{\sqrt{t_{i+1}}}{f\left(t_{i}\right)}\right\}
$$

причем мы принимаем $\inf \varnothing:=\infty$. Тогда

$$
\begin{aligned}
\left\{\sigma_{i} \leqslant t_{i+1}\right\} & \cap\left\{\left\|\mathbf{M}\left(\cdot, t_{i+1}\right)-\mathbf{M}(\cdot, \sigma)\right\| \leqslant \sqrt{t_{i+1}-t_{i}}\right\} \\
& \subset\left\{\left\|\mathbf{M}\left(\cdot, t_{i+1}\right)\right\| \leqslant \frac{\sqrt{t_{i+1}}}{f\left(t_{i}\right)}+\sqrt{t_{i+1}-t_{i}}\right\} .
\end{aligned}
$$


Рассмотрим фильтрацию, порожденную вторым параметром:

$$
\mathscr{F}_{t}:=\sigma\{\mathbf{M}(s, v): s \in[0,1], v \in[0, t]\}, \quad t \geqslant 0 .
$$

Согласно строго марковскому свойству, в пределах события $\left\{\sigma_{i} \leqslant t_{i+1}\right\}$ мы имеем

$$
\begin{aligned}
& \mathbf{P}\left\{\left\|\mathbf{M}\left(\cdot, t_{i+1}\right)-\mathbf{M}(\cdot, \sigma)\right\| \leqslant \sqrt{t_{i+1}-t_{i}} \mid \mathscr{F}_{\sigma_{i}}\right\} \\
& \quad \geqslant \mathbf{P}\{\|\mathbf{M}(\cdot, 1)\| \leqslant 1\}:=c_{1}>0,
\end{aligned}
$$

поэтому

$$
\begin{aligned}
\mathbf{P}\left\{\sigma_{i} \leqslant t_{i+1}\right\} & \leqslant \frac{1}{c_{1}} \mathbf{P}\left\{\left\|\mathbf{M}\left(\cdot, t_{i+1}\right)\right\| \leqslant \frac{\sqrt{t_{i+1}}}{f\left(t_{i}\right)}+\sqrt{t_{i+1}-t_{i}}\right\} \\
& =\frac{1}{c_{1}} \mathbf{P}\left\{\|\mathbf{M}(\cdot, 1)\| \leqslant \frac{1}{f\left(t_{i}\right)}+\frac{\sqrt{t_{i+1}-t_{i}}}{\sqrt{t_{i+1}}}\right\} \\
& \leqslant \frac{1}{c_{1}} \mathbf{P}\left\{\|\mathbf{M}(\cdot, 1)\| \leqslant \frac{1}{f\left(t_{i}\right)}+\frac{c_{2}}{f^{3}\left(t_{i}\right)}\right\},
\end{aligned}
$$

где последнее неравенство вытекает из (2.5). Поэтому, с учетом (1.3), мы получаем

$\mathbf{P}\left\{\sigma_{i} \leqslant t_{i+1}\right\} \leqslant c_{3} \exp \left(-\frac{\pi^{2}}{8\left[1 / f\left(t_{i}\right)+c_{2} / f^{3}\left(t_{i}\right)\right]^{2}}\right) \leqslant c_{4} \exp \left(-\frac{\pi^{2}}{8} f^{2}\left(t_{i}\right)\right)$.

Согласно (2.6), отсюда следует $\sum_{i} \mathbf{P}\left\{\sigma_{i} \leqslant t_{i+1}\right\}<\infty$. Поскольку

$$
\left\{\sigma_{i} \leqslant t_{i+1}\right\}=\left\{\inf _{t \in\left[t_{i}, t_{i+1}\right]}\|\mathbf{M}(\cdot, t)\| \leqslant \frac{\sqrt{t_{i+1}}}{f\left(t_{i}\right)}\right\},
$$

то лемма Бореля-Кантелли обеспечивает, что п.н. при всех достаточно больших $i$ верно inf $\operatorname{t\in [t}_{\left.t, t_{i+1}\right]}\|\mathbf{M}(\cdot, t)\|>\sqrt{t_{i+1}} \cdot f\left(t_{i}\right)$. Следовательно, для всех $t \in\left[t_{i}, t_{i+1}\right]$ (напомним, что функция $f-$ неубывающая) справедлива цепочка неравенств

$$
\|\mathbf{M}(\cdot, t)\|>\frac{\sqrt{t_{i+1}}}{f\left(t_{i}\right)} \geqslant \frac{\sqrt{t}}{f(t)} .
$$

Эта оценка завершает доказательство соотношения (2.1).

Прежде чем заниматься непосредственно соотношением (2.2), мы введем следующее обозначение и сформулируем одну вспомогательную вероятностную оценку. Для любой пары случайных процессов $V_{1}$ и $V_{2} \mathrm{c}$ траекториями из $C[0,1]$ и любых $\varepsilon>0$ и $\tau \in(0,1]$ обозначим

$$
D\left(V_{1}, V_{2}, \varepsilon, \tau\right):=\left\{\left\|V_{1}\right\| \leqslant \varepsilon,\left\|\sqrt{1-\tau} V_{1}+\sqrt{\tau} V_{2}\right\| \leqslant \varepsilon\right\} .
$$


Предложение 2.1. Пусть $W_{1} u W_{2}$ - независимье одномернье броуновские движения. Тогда для любых $\varepsilon>0 u \tau \in(0,1]$ верно

$$
\mathbf{P}\left\{D\left(W_{1}, W_{2}, \varepsilon, \tau\right)\right\} \leqslant c_{5} \exp \left(-\frac{\pi^{2}}{8 \varepsilon^{2}}-\frac{c_{6} \tau^{1 / 3}}{\varepsilon^{2}}\right) .
$$

С геометрической точки зрения в этом предложении идет речь о вероятности того, что броуновское движение длительное время остается внутри вытянутого параллелограмма. Особо отметим важность показателя степени $\frac{1}{3}$ параметра $\tau$ в правой части. Дело в том, что прямолинейное применение неравенства Андерсона дало бы оценку со степенью 1, увы, не достаточную для доказательства соотношения (2.2).

Принимая пока на веру справедливость предложения 2.1 (его доказательству посвящен специальный раздел 3), мы готовы начать вычисления.

Д ок аз а т ель с т в о с о о т н оше н и я $(2.2)$. Предположим, что $\int^{\infty}\left(f^{6}(t) / t\right) \exp \left(-\left(\pi^{2} / 8\right) f^{2}(t)\right) d t=\infty$, и рассмотрим последовательность событий

$$
A_{i}:=\left\{\left\|\mathbf{M}\left(\cdot, t_{i}\right)\right\| \leqslant \frac{\sqrt{t_{i}}}{f\left(t_{i}\right)}\right\}, \quad i \geqslant i_{0},
$$

при достаточно больших значениях индекса $i_{0}$.

Из (1.3) следует, что

$$
\mathbf{P}\left(A_{i}\right) \asymp \exp \left(-\frac{\pi^{2}}{8} f^{2}\left(t_{i}\right)\right),
$$

откуда с помощью (2.6) получаем, что $\sum_{i} \mathbf{P}\left(A_{i}\right)=\infty$.

$\mathrm{C}$ целью применения одной из версий леммы Бореля-Кантелли мы исследуем двойную сумму $\sum \sum_{i<j \leqslant N} \mathbf{P}\left(A_{i} A_{j}\right)$ и оценим ее сверху величиной, кратной выражению $\left[\sum_{i \leqslant N} \mathbf{P}\left(A_{j}\right)\right]^{2}$. Желаемая оценка для $\mathbf{P}\left(A_{i} A_{j}\right)$ будет получена тремя различными способами, в зависимости от расположения пары $(i, j)$. Обозначим

$$
\begin{aligned}
& E_{1}=E_{1}(N):=\left\{(i, j): i_{0} \leqslant i<j \leqslant N, j-i \leqslant(\ln i)^{3}\right\} \\
& E_{2}=E_{2}(N):=\left\{(i, j): i_{0} \leqslant i<j \leqslant N,(\ln i)^{3}<j-i \leqslant(\ln i)^{4}\right\}, \\
& E_{3}=E_{3}(N):=\left\{(i, j): i_{0} \leqslant i<j \leqslant N, j-i>(\ln i)^{4}\right\} .
\end{aligned}
$$

Начнем с тех более простых случаев, где классическое неравенство Андерсона (см., например, [2, гл. 11]) дает удовлетворительную оценку: так как $\left(t_{j}-t_{i}\right)^{-1 / 2}\left[\mathbf{M}\left(\cdot, t_{j}\right)-\mathbf{M}\left(\cdot, t_{i}\right)\right]$ и $t_{i}^{-1 / 2} \mathbf{M}\left(\cdot, t_{i}\right)$ являются независимыми броуновскими движениями, мы имеем

$$
\begin{aligned}
\mathbf{P}\left(A_{i} A_{j}\right) & \leqslant \mathbf{P}\left(A_{i}\right) \mathbf{P}\left\{\left\|\mathbf{M}\left(\cdot, t_{j}-t_{i}\right)\right\| \leqslant \frac{\sqrt{t_{j}}}{f\left(t_{j}\right)}\right\} \\
& =\mathbf{P}\left(A_{i}\right) \mathbf{P}\left\{\|\mathbf{M}(\cdot, 1)\| \leqslant \frac{1}{\sqrt{1-\left(t_{i} / t_{j}\right)} f\left(t_{j}\right)}\right\} .
\end{aligned}
$$


Если $(i, j) \in E_{3}$, то $j-i>\frac{1}{2}(\ln j)^{4}$, что в силу $(2.3)$ влечет $t_{i} / t_{j} \leqslant 1 / f^{2}\left(t_{j}\right)$; следовательно, из (1.3) вытекает

$$
\begin{aligned}
\mathbf{P}\left(A_{i} A_{j}\right) & \leqslant \mathbf{P}\left(A_{i}\right) \mathbf{P}\left\{\|\mathbf{M}(\cdot, 1)\| \leqslant \frac{1}{\sqrt{1-f^{-2}\left(t_{j}\right)} f\left(t_{j}\right)}\right\} \\
& \leqslant c_{7} \mathbf{P}\left(A_{i}\right) \exp \left(-\frac{\pi^{2}\left(1-f^{-2}\left(t_{j}\right)\right)}{8} f^{2}\left(t_{j}\right)\right) \\
& \leqslant c_{8} \mathbf{P}\left(A_{i}\right) \exp \left(-\frac{\pi^{2}}{8} f^{2}\left(t_{j}\right)\right) \leqslant c_{9} \mathbf{P}\left(A_{i}\right) \mathbf{P}\left(A_{j}\right) .
\end{aligned}
$$

Поэтому

$$
\sum_{(i, j) \in E_{3}} \mathbf{P}\left(A_{i} A_{j}\right) \leqslant c_{10}\left(\sum_{i \leqslant N} \mathbf{P}\left(A_{i}\right)\right)^{2} .
$$

Теперь рассмотрим случай $(i, j) \in E_{2}$. Неравенство $j-i>(\ln i)^{3}$ обеспечивает $t_{i} / t_{j} \leqslant c_{11}<1$. Используя $(2.8),(2.3)$ и (1.3), мы находим

$$
\mathbf{P}\left(A_{i} A_{j}\right) \leqslant \mathbf{P}\left(A_{i}\right) \mathbf{P}\left\{\|\mathbf{M}(\cdot, 1)\| \leqslant \frac{1}{c_{12} \sqrt{\ln j}}\right\} \leqslant c_{13} \mathbf{P}\left(A_{i}\right) j^{-c_{14}}
$$

Соответственно,

$$
\sum_{(i, j) \in E_{2}} \mathbf{P}\left(A_{i} A_{j}\right) \leqslant c_{13} \sum_{i \leqslant N} \mathbf{P}\left(A_{i}\right) \sum_{j: i<j \leqslant i+(\ln i)^{4}} j^{-c_{14}} \leqslant c_{15} \sum_{i \leqslant N} \mathbf{P}\left(A_{i}\right) .
$$

Перейдем теперь к получению верхних оценок для $\mathbf{P}\left(A_{i} A_{j}\right)$ в трудном случае $(i, j) \in E_{1}$. Прямое применение неравенства Андерсона здесь оказывается слишком грубым приемом. Именно здесь нам пригодится предложение 2.1. В самом деле, обозначая, как и раньше, $W_{1}$ и $W_{2}$ пару одномерных броуновских движений, мы имеем

$$
\begin{aligned}
\mathbf{P}\left(A_{i} A_{j}\right) & =\mathbf{P}\left\{\left\|W_{1}\right\| \leqslant \frac{1}{f\left(t_{i}\right)},\left\|\sqrt{1-\tau} W_{1}+\sqrt{\tau} W_{2}\right\| \leqslant \frac{1}{f\left(t_{j}\right)}\right\} \\
& \leqslant \mathbf{P}\left\{\left\|W_{1}\right\| \leqslant \frac{1}{f\left(t_{i}\right)},\left\|\sqrt{1-\tau} W_{1}+\sqrt{\tau} W_{2}\right\| \leqslant \frac{1}{f\left(t_{i}\right)}\right\},
\end{aligned}
$$

где $\tau=\tau_{i j}:=1-\left(t_{i} / t_{j}\right)$. Если $(i, j) \in E_{1}$, то, вновь используя (2.3), мы выводим оценку

$$
\tau \geqslant c_{16} \frac{j-i}{(\ln i)^{3}} \geqslant \frac{c_{17}(j-i)}{f^{6}\left(t_{i}\right)} .
$$

Применим предложение 2.1 к $\varepsilon:=1 / f\left(t_{i}\right)$ и получим (используя $(2.7)$ на последнем шаге)

$$
\begin{aligned}
\mathbf{P}\left(A_{i} A_{j}\right) & \leqslant c_{5} \exp \left(-\frac{\pi^{2} f\left(t_{i}\right)}{8}-c_{18}(j-i)^{1 / 3}\right) \\
& \leqslant c_{19} \mathbf{P}\left(A_{i}\right) \exp \left(-c_{18}(j-i)^{1 / 3}\right)
\end{aligned}
$$


Следовательно,

$$
\sum_{(i, j) \in E_{1}} \mathbf{P}\left(A_{i} A_{j}\right) \leqslant c_{19} \sum_{i \leqslant N} \mathbf{P}\left(A_{i}\right) \sum_{j>i} \exp \left(-c_{18}(j-i)^{1 / 3}\right) \leqslant c_{20} \sum_{i \leqslant N} \mathbf{P}\left(A_{i}\right) .
$$

Комбинируя эту оценку с (2.9) и (2.10), мы получаем:

$$
\limsup _{N \rightarrow \infty} \frac{\sum \sum_{i_{0} \leqslant i, j \leqslant N} \mathbf{P}\left(A_{i} A_{j}\right)}{\left[\sum_{i_{0} \leqslant i \leqslant N} \mathbf{P}\left(A_{i}\right)\right]^{2}} \leqslant c_{10}<\infty .
$$

Далее мы воспользуемся следующим обобщением леммы БореляКантелли.

Лемма 2.1 (Кочен и Стоун [14]). Пусть $\left(A_{i}\right)$ - такая последовательность событий, что $\sum_{i} \mathbf{P}\left(A_{i}\right)=\infty$. Тогда

$$
\mathbf{P}\left\{A_{i} \text { б. и. }\right\} \geqslant\left[\liminf _{N \rightarrow \infty} \frac{\sum \sum_{i_{0} \leqslant i, j \leqslant N} \mathbf{P}\left(A_{i} A_{j}\right)}{\left[\sum_{i_{0} \leqslant i \leqslant N} \mathbf{P}\left(A_{i}\right)\right]^{2}}\right]^{-1} .
$$

Согласно лемме 2.1 , мы имеем

$$
\mathbf{P}\left\{\left\|\mathbf{M}\left(\cdot, t_{i}\right)\right\| \leqslant \frac{\sqrt{t_{i}}}{f\left(t_{i}\right)} \text { б.ч. }\right\}>0 .
$$

Вместо подпоследовательности $t_{i}=\exp \left(i /(\ln i)^{3}\right)$, определенной в (2.4), можно рассматривать подпоследовательность $\hat{t}_{i}=\left\lfloor\exp \left(i /(\ln i)^{3}\right)\right\rfloor$. Поскольку $\hat{t}_{i} \leqslant t_{i} \leqslant \hat{t}_{i}+1$, то аргументы, ведущие к $(2.11)$, применимы и к $\left(\hat{t}_{i}\right)$ вместо $\left(t_{i}\right)$ с точностью до замены некоторых констант. Соответственно, мы имеем

$$
\mathbf{P}\left\{\left\|\mathbf{M}\left(\cdot, \hat{t}_{i}\right)\right\| \leqslant \frac{\sqrt{\hat{t}_{i}}}{f\left(\hat{t}_{i}\right)} \text { б.ч. }\right\}>0,
$$

что, в свою очередь, влечет

$$
\mathbf{P}\left\{\|\mathbf{M}(\cdot, n)\| \leqslant \frac{\sqrt{n}}{f(n)} \text { б.ч. }\right\}>0 .
$$

Отметим, что $\mathbf{M}(\cdot, n)=\sum_{k=1}^{n}[\mathbf{M}(\cdot, k)-\mathbf{M}(\cdot, k-1)]$ и что $\{\mathbf{M}(\cdot, k)-\mathbf{M}(\cdot, k-1), k \geqslant 1\}$ является последовательностью независимых одинаково распределенных случайных элементов пространства $(C([0,1], \mathbf{R}),\|\cdot\|)$. Поскольку любая конечная перестановка $\{\mathbf{M}(\cdot, k)-$ $\mathbf{M}(\cdot, k-1), k \geqslant 1\}$ не изменяет события $\{\|\mathbf{M}(\cdot, n)\| \leqslant \sqrt{n} / f(n)$ б.ч. $\}$, то закон 0-1 Хьюитта-Сэвиджа гарантирует, что

$$
\mathbf{P}\left\{\|\mathbf{M}(\cdot, n)\| \leqslant \frac{\sqrt{n}}{f(n)} \text { б.ч. }\right\}=1 .
$$

Таким образом, (2.2) полностью доказано. 
3. Доказательство предложения 2.1. Этот раздел посвящен доказательству предложения 2.1. Напомним его суть: пусть $W_{1}$ и $W_{2}-$ два независимых броуновских движения, тогда существуют такие конечные положительные постоянные $c_{5}$ и $c_{6}$, что для всех $\varepsilon>0$ и $\tau \in(0,1]$ верно

$$
\begin{aligned}
& \mathbf{P}\left\{\left|W_{1}(t)\right| \leqslant \varepsilon,\left|\sqrt{1-\tau} W_{1}(t)+\sqrt{\tau} W_{2}(t)\right| \leqslant \varepsilon \text { для всех } t \in[0,1]\right\} \\
& \quad \leqslant c_{5} \exp \left(-\frac{\pi^{2}}{8 \varepsilon^{2}}-\frac{c_{6} \tau^{1 / 3}}{\varepsilon^{2}}\right) .
\end{aligned}
$$

Для произвольного $h>0$ зададим последовательность моментов остановки $\theta_{k}=\theta_{k}(h)$, зависящую только от процесса $W_{2}$, полагая $\theta_{0}=0$, а затем

$$
\begin{aligned}
& \theta_{2 k+1}:=\inf \left\{t>\theta_{2 k}:\left|W_{2}(t)\right|=2 h\right\}, \\
& \theta_{2 k+2}:=\inf \left\{t>\theta_{2 k+1}:\left|W_{2}(t)\right|=h\right\}, \quad k \geqslant 0 .
\end{aligned}
$$

Обозначим также $\theta_{k}^{\prime}:=\min \left\{\theta_{k}, 1\right\}$. Далее, определим длительность пересечения полосы снизу вверх

$$
Y_{k}(h):=\theta_{2 k+1}-\theta_{2 k} \quad(k \geqslant 0)
$$

и длительность пересечения сверху вниз

$$
Z_{k}(h):=\theta_{2 k}-\theta_{2 k-1} \quad(k \geqslant 1) .
$$

Аналогично, $Y_{k}^{\prime}(h)$ и $Z_{k}^{\prime}(h)$ определяются при помощи $\theta_{k}^{\prime}$.

Отметим некоторые моментные свойства и свойства самоподобия последовательностей $Y_{k}(h)$ и $Z_{k}(h)$. Для длительностей пересечения снизу вверх мы имеем $Y_{k}(h) \stackrel{\mathrm{d}}{=} h^{2} Y_{k}(1)$. Величины $\left\{Y_{k}(1), k \geqslant 0\right\}$ независимы и одинаково распределены при $k \geqslant 1$. Хорошо известно, что некоторый экспоненциальный момент, например $\mathbf{E} \exp \left\{Y_{k}(1) / 4\right\}$, конечен. По экспоненциальному неравенству Чебышева для каждого $b>\mathbf{E} Y_{0}(1)$ существует такая положительная постоянная $c_{b}$, что для всех $h$ и всех целых $N$ верно

$$
\mathbf{P}\left\{\sum_{k=0}^{N-1} Y_{k}(h) \geqslant b h^{2} N\right\} \leqslant \exp \left\{-c_{b} N\right\} .
$$

$\mathrm{C}$ другой стороны, для длительностей пересечения сверху вниз мы имеем не только свойство самоподобия $Z_{k}(h) \stackrel{\mathrm{d}}{=} h^{2} Z_{k}(1)$ для независимых одинаково распределенных величин $Z_{k}(h)$, но еще и устойчивость порядка $\frac{1}{2}$. Именно,

$$
\sum_{k=1}^{N} Z_{k}(1) \stackrel{\mathrm{d}}{=} Z_{1}(N) \stackrel{\mathrm{d}}{=} N^{2} Z_{1}(1) .
$$


В частности, мы имеем

$$
\begin{aligned}
\mathbf{P}\left\{\sum_{k=1}^{N} Z_{k}(h) \leqslant 1\right\} & =\mathbf{P}\left\{N^{2} h^{2} Z_{1}(1) \leqslant 1\right\}=\mathbf{P}\left\{\sup _{0 \leqslant t \leqslant N^{-2} h^{-2}} W_{2}(t) \geqslant 1\right\} \\
& \leqslant 2 \exp \left\{-\frac{N^{2} h^{2}}{2}\right\} .
\end{aligned}
$$

При заданных $N$ и $h$ рассмотрим следующие события:

$$
\begin{array}{rlrl}
D_{1} & :=\left\{\left\|W_{1}\right\| \leqslant \varepsilon\right\} ; & D_{2,1}:=\left\{\sum_{k=0}^{N-1} Y_{k}(h) \geqslant b h^{2} N\right\} ; \\
D_{2,2}:=\left\{\sum_{k=1}^{N} Z_{k}(h) \leqslant 1\right\} ; & D_{2,3}:=\left(D_{2,1} \cup D_{2,2}\right)^{c} .
\end{array}
$$

Обозначим для краткости $D:=D\left(W_{1}, W_{2}, \varepsilon, \tau\right)$. Поскольку $D \subset D_{1}$, то мы имеем

$$
D=\left(D D_{2,1}\right) \cup\left(D D_{2,2}\right) \cup\left(D D_{2,3}\right) \subset\left(D_{1} D_{2,1}\right) \cup\left(D_{1} D_{2,2}\right) \cup\left(D D_{2,3}\right) .
$$

Используя оценки $(3.1),(3.2)$ и независимость $W_{1}$ и $W_{2}$, получаем

$$
\mathbf{P}\left\{\left(D_{1} D_{2,1}\right) \cup\left(D_{1} D_{2,2}\right)\right\} \leqslant \mathbf{P}\left(D_{1}\right)\left(\exp \left\{-c_{b} N\right\}+2 \exp \left\{\frac{-N^{2} h^{2}}{2}\right\}\right) .
$$

Рассмотрим теперь траектории из $D D_{2,3}$. Отметим, что в этом случае верно

$$
\sum_{k=0}^{N-1} Y_{k}(h) \leqslant b h^{2} N
$$

и $\sum_{k=1}^{N} Z_{k}(h)>1$. Следовательно, $\theta_{2 N}>1$, и мы имеем

$$
\sum_{k=0}^{N-1} Y_{k}^{\prime}(h)+\sum_{k=1}^{N} Z_{k}^{\prime}(h)=1
$$

Ключевым является следующий факт: для каждой траектории из $D D_{2,3}$ верно

$$
W_{1}(t) \in \begin{cases}{[-\varepsilon, \varepsilon],} & \text { если } t \in \bigcup_{k=0}^{N-1}\left[\theta_{2 k}^{\prime}, \theta_{2 k+1}^{\prime}\right], \\ {\left[-\varepsilon,-\varepsilon+2 \varepsilon_{h}\right] \operatorname{sign}\left\{W_{2}\left(\theta_{2 k-1}\right)\right\},} & \text { если } t \in \bigcup_{k=1}^{N}\left[\theta_{2 k-1}^{\prime}, \theta_{2 k}^{\prime}\right],\end{cases}
$$

где $\varepsilon_{h}$ обозначает половину ширины интервала, в котором процесс $W_{1}$ обязан находиться, когда $W_{2}$ достигает уровня $h$. Далее, при $h>2 \sqrt{\tau} \varepsilon$ 
этот параметр удовлетворяет соотношениям

$$
\varepsilon_{h}:=\left(\varepsilon-\frac{\sqrt{\tau}}{2 \sqrt{1-\tau}}\left(h-\frac{\sqrt{\tau} \varepsilon}{1+\sqrt{1-\tau}}\right)\right)_{+} \leqslant \varepsilon-\frac{\sqrt{\tau} h}{4},
$$

откуда

$$
\varepsilon_{h}^{-2} \geqslant \varepsilon^{-2}\left(1-\frac{\sqrt{\tau} h}{4 \varepsilon}\right)^{-2} \geqslant \varepsilon^{-2}+\frac{\sqrt{\tau} h}{2 \varepsilon^{3}} .
$$

Следовательно, по неравенству Андерсона и оценке малых уклонений (1.3) для $W_{1}$ мы имеем

$$
\mathbf{P}\left\{D D_{2,3} \mid W_{2}\right\} \leqslant\left(\frac{4}{\pi}\right)^{2 N} \prod_{k=0}^{N-1} \exp \left\{-\frac{\pi^{2}}{8 \varepsilon^{2}} Y_{k}^{\prime}(h)\right\} \prod_{k=1}^{N} \exp \left\{-\frac{\pi^{2}}{8 \varepsilon_{h}^{2}} Z_{k}^{\prime}(h)\right\}
$$

в то время как из (3.5) и (3.6) следует

$$
\begin{gathered}
\varepsilon^{-2} \sum_{k=0}^{N-1} Y_{k}^{\prime}(h)+\varepsilon_{h}^{-2} \sum_{k=1}^{N} Z_{k}^{\prime}(h) \geqslant \varepsilon^{-2}\left(\sum_{k=0}^{N-1} Y_{k}^{\prime}(h)+\sum_{k=1}^{N} Z_{k}^{\prime}(h)\right) \\
+\frac{\sqrt{\tau} h}{2 \varepsilon^{3}} \sum_{k=1}^{N} Z_{k}^{\prime}(h)=\varepsilon^{-2}+\frac{\sqrt{\tau} h}{2 \varepsilon^{3}}\left(1-\sum_{k=0}^{N-1} Y_{k}^{\prime}(h)\right) .
\end{gathered}
$$

Заменим $\sum_{k=0}^{N-1} Y_{k}^{\prime}(h)$ большей суммой $\sum_{k=0}^{N-1} Y_{k}(h)$ и применим (3.4). Таким способом получается равномерная оценка для условной вероятности

$$
\mathbf{P}\left(D D_{2,3} \mid W_{2}\right) \leqslant \mathbf{1}_{D_{2,3}}\left(\frac{4}{\pi}\right)^{2 N} \exp \left\{-\frac{\pi^{2}}{8 \varepsilon^{2}}-\frac{\pi^{2}}{16 \varepsilon^{3}} \sqrt{\tau} h\left(1-b h^{2} N\right)\right\},
$$

и мы получаем неравенство

$$
\mathbf{P}\left(D D_{2,3}\right) \leqslant\left(\frac{4}{\pi}\right)^{2 N} \exp \left\{-\frac{\pi^{2}}{8 \varepsilon^{2}}-\frac{\pi^{2}}{16 \varepsilon^{3}} \sqrt{\tau} h\left(1-b h^{2} N\right)\right\} .
$$

Теперь мы окончательно определим параметры конструкции. Пусть

$$
h:=2 \varepsilon \tau^{-1 / 6} ; \quad b:=\max \left\{\frac{4 \ln (4 / \pi)}{\pi^{2}}, \mathbf{E} Y_{0}(1)\right\}+1 ; \quad N:=\left\lfloor\frac{1}{2 b h^{2}}\right\rfloor .
$$

Рассмотрим два случая. Если $2 b h^{2} \geqslant 1$, то $\varepsilon^{-2} \tau^{1 / 3}=4 h^{-2} \leqslant 8 b$, и утверждение предложения 2.1 тривиально, так как

$$
\mathbf{P}(D) \leqslant \mathbf{P}\left(D_{1}\right) \leqslant \frac{4}{\pi} \exp \left\{-\frac{\pi^{2}}{8 \varepsilon^{2}}\right\} \leqslant \frac{4 e}{\pi} \exp \left\{-\frac{\pi^{2}}{8 \varepsilon^{2}}-\frac{\tau^{1 / 3}}{8 b \varepsilon^{2}}\right\} .
$$

С другой стороны, если $2 b h^{2} \leqslant 1$, то из (3.7) извлекается неравенство

$$
\mathbf{P}\left(D D_{2,3}\right) \leqslant \exp \left\{-\frac{\pi^{2}}{8 \varepsilon^{2}}-\left(\frac{\pi^{2}}{16}-\frac{\ln (4 / \pi)}{4 b}\right) \frac{\tau^{1 / 3}}{\varepsilon^{2}}\right\} .
$$

Предложение 2.1, таким образом, вытекает из совместного применения последней оценки и (3.3). 
4. Применение к эмпирическим процессам. Пусть $U_{1}, U_{2}, \ldots$ последовательность независимых случайных величин, равномерно распределенных в интервале $(0,1)$. Пусть

$$
\alpha_{n}(s):=n^{1 / 2}\left(\sum_{i=1}^{n} \mathbf{1}_{\left\{U_{i} \leqslant s\right\}}-s\right), \quad s \in[0,1]
$$

- равномерный эмпирический процесс, построенный по первым $n$ наблюдениям. Как и ранее, мы работаем с равномерной нормой $\|f\|:=$ $\sup _{s \in[0,1]}|f(s)|$.

Нашей целью является доказательство теоремы 1.2 , характеризующей нижние функции эмпирического процесса. В качестве первого шага в этом направлении мы сначала получим соответствующий гауссовский результат для процесса Кифера $\left\{K_{n}(s) ; n \geqslant 0, s \in[0,1]\right\}$. По определению, процесс Кифера это центрированное гауссовское поле с ковариацией

$$
\mathbf{E}\left[K_{n}(s) K_{m}(u)\right]:=\min \{n, m\}(\min \{s, u\}-s u) .
$$

Для него аналог теоремы 1.2 выглядит следующим образом.

Теорема 4.1. Пусть $K_{n}(s)$ - прочесс Кифера. Тогда для любой положительной возрастающей последовательности $\left(\lambda_{n}\right)$ верно

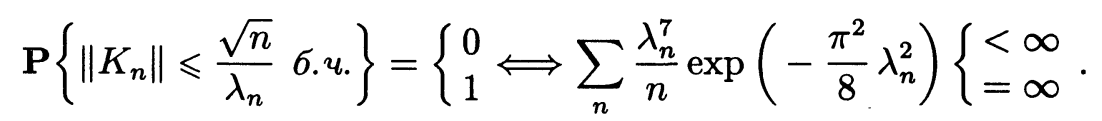

В доказательстве теоремы 1.1 мы уже объясняли, что достаточно изучать «критические функции» в смысле (2.3). По тем же причинам в доказательстве теорем 1.2 и 4.1 мы можем, не ограничивая общности, считать, что

$$
\frac{\sqrt{\ln \ln n}}{2} \leqslant \lambda_{n} \leqslant \sqrt{\ln \ln n} \quad \text { для } \quad n \geqslant n_{0} .
$$

При этом предположении эквивалентность теорем 1.2 и 4.1 очевидна. В самом деле, теорема Комлоша, Майора и Тушнади о сильной аппроксимации [16] обеспечивает возможность такого совместного построения $\alpha_{n}$ и $K_{n}$ на общем вероятностном пространстве, при котором

$$
\left\|\alpha_{n}-\frac{K_{n}}{\sqrt{n}}\right\|=o\left(\frac{\ln ^{2} n}{n^{1 / 2}}\right)=o\left(\lambda_{n}^{-3}\right)=o\left(\left|\frac{1}{\lambda_{n}}-\frac{1}{\lambda_{n} \pm 1 / \lambda_{n}}\right|\right)
$$

почти наверное, в то время как ряд из правой части нашего теста сходится (или расходйтся) одновременно для последовательностей $\lambda_{n}$ и $\lambda_{n} \pm 1 / \lambda_{n}$

Доказательство теоремы 4.1 проводится совершенно в том же духе, что и доказательство теоремы 1.1 , так что большую часть рассуждений 
мы позволим себе опустить. Нужны лишь две поправки. Вместо применения асимптотики (1.3) для равномерной нормы броуновского движения мы воспользуемся соответствующим результатом для броуновского моста (поскольку $K_{1}$ это как раз стандартный броуновский мост):

$$
\mathbf{P}\left\{\left\|K_{1}\right\| \leqslant \varepsilon\right\} \sim \frac{\sqrt{2 \pi}}{\varepsilon} \exp \left(-\frac{\pi^{2}}{8 \varepsilon^{2}}\right), \quad \varepsilon \rightarrow 0 .
$$

(Вычисление распределения величины $\left\|K_{1}\right\|$ восходит по меньшей мере к работе А. Н. Колмогорова [15].) С другой стороны, аналогом предложения 2.1 будет служить следующий результат. Напомним введенное ранее обозначение

$$
D\left(V_{1}, V_{2}, \varepsilon, \tau\right):=\left\{\left\|V_{1}\right\| \leqslant \varepsilon,\left\|\sqrt{1-\tau} V_{1}+\sqrt{\tau} V_{2}\right\| \leqslant \varepsilon\right\}
$$

для любых случайных процессов $V_{1}$ и $V_{2}$.

Предложение 4.1. (i) Если $\stackrel{\circ}{W}_{1}-$ стандартный броуновский мост, не зависящий от броуновского движения $W_{2}$, то для любых $\varepsilon \in(0,1] u \tau \in(0,1]$ верно

$$
\mathbf{P}\left\{D\left(\stackrel{\circ}{W}_{1}, W_{2}, \varepsilon, \tau\right)\right\} \leqslant \frac{c_{21}}{\varepsilon} \exp \left(-\frac{\pi^{2}}{8 \varepsilon^{2}}-\frac{c_{22} \tau^{1 / 3}}{\varepsilon^{2}}\right) .
$$

(ii) Пусть $\stackrel{\circ}{W}_{1}$ и $\stackrel{\circ}{W}_{2}$ - независимые броуновские мосты. Тогда для любых $\varepsilon \in(0,1] u \tau \in(0,1]$ верно

$$
\mathbf{P}\left\{D\left(\stackrel{\circ}{W}_{1}, \stackrel{\circ}{W}_{2}, \varepsilon, \tau\right)\right\} \leqslant c_{23} \max \left\{\frac{1}{\varepsilon}, \frac{\sqrt{\tau}}{\varepsilon^{4}}\right\} \exp \left(-\frac{\pi^{2}}{8 \varepsilon^{2}}-\frac{c_{24} \tau^{1 / 3}}{\varepsilon^{2}}\right) .
$$

Хотя часть (i) данного предложения и не требуется непосредственно для доказательства теоремы 4.1, она нужна для обоснования части (ii).

Если считать предложение 4.1 доказанным, то доказательство теоремы 4.1 буквально следует доказательству теоремы 1.1, так что мы не будем повторяться. Оставшаяся часть раздела посвящена выводу предложения 4.1 .

Д оказ ат ель с тв о п редл о жен ия 4.1. Начнем с доказательства соотношения (4.3). Положим $q:=1-\varepsilon^{2}$ и будем для краткости писать

$$
\begin{gathered}
\|v(\cdot)\|_{[0, q]}:=\sup _{0 \leqslant t \leqslant q}|v(t)|, \\
D_{q}\left(V_{1}, V_{2}, \varepsilon, \tau\right):=\left\{\left\|V_{1}\right\|_{[0, q]} \leqslant \varepsilon,\left\|\sqrt{1-\tau} V_{1}+\sqrt{\tau} V_{2}\right\|_{[0, q]} \leqslant \varepsilon\right\} .
\end{gathered}
$$

По формуле полной вероятности имеем

$$
\begin{aligned}
\mathbf{P} & \left\{D_{q}\left(W_{1}, W_{2}, \varepsilon, \tau\right)\right\} \\
& =\int_{-\infty}^{\infty} \mathbf{P}\left\{D_{q}\left(W_{1}, W_{2}, \varepsilon, \tau\right) \mid W_{1}(1)=r\right\} \frac{\exp \left\{-r^{2} / 2\right\}}{\sqrt{2 \pi}} d r
\end{aligned}
$$




$$
\begin{aligned}
& \geqslant c_{25} \varepsilon \inf _{|r| \leqslant \varepsilon / 2} \mathbf{P}\left\{D_{q}\left(W_{1}, W_{2}, \varepsilon, \tau\right) \mid W_{1}(1)=r\right\} \\
& =c_{25} \varepsilon \inf _{|r| \leqslant \varepsilon / 2} \mathbf{P}\left\{D_{q}\left(\stackrel{\circ}{W_{1}}+r \mathrm{Id}, W_{2}, \varepsilon, \tau\right)\right\} \\
& =c_{25} \varepsilon \inf _{|r| \leqslant \varepsilon / 2} \mathbf{P}\left\{D_{q}\left(\stackrel{\circ}{W}_{1}+r h_{q}, W_{2}, \varepsilon, \tau\right)\right\},
\end{aligned}
$$

где

$$
h_{q}(t):=\left\{\begin{array}{lll}
t & \text { для } & 0 \leqslant t \leqslant q, \\
q(1-t) \varepsilon^{-2} & \text { для } & q \leqslant t \leqslant 1
\end{array}\right.
$$

является допустимым сдвигом броуновского моста, причем плотность соответствующей сдвинутой меры по формуле Камерона-Мартина имеет вид

$$
p_{r h_{q}}(v)=\exp \left\{-\frac{r^{2} q}{2 \varepsilon^{2}}+\frac{r}{\varepsilon^{2}} v(q)\right\}
$$

Стоит заметить, что при выполнении условий $|r| \leqslant \varepsilon / 2$ и $\left\|\stackrel{\circ}{W}_{1}\right\|_{[0, q]} \leqslant \varepsilon$ мы имеем

$$
p_{r h_{q}}\left(\stackrel{\circ}{W}_{1}\right) \geqslant \exp \left\{-\frac{1}{8}-\frac{\left|\stackrel{\circ}{W}_{1}(q)\right|}{4 \varepsilon}\right\} \geqslant e^{-3 / 8} .
$$

Находим по формуле Камерона-Мартина

$$
\begin{gathered}
\mathbf{P}\left\{D_{q}\left(\stackrel{\circ}{W}_{1}+r h_{q}, W_{2}, \varepsilon, \tau\right)\right\}=\mathbf{E}\left[\mathbf{1}_{D_{q}\left(\stackrel{\circ}{W}_{1}, W_{2}, \varepsilon, \tau\right)} p_{r h_{q}}\left(\stackrel{\circ}{W}_{1}\right)\right] \\
\geqslant e^{-3 / 8} \mathbf{P}\left\{D_{q}\left(\stackrel{\circ}{W}_{1}, W_{2}, \varepsilon, \tau\right)\right\} \geqslant e^{-3 / 8} \mathbf{P}\left\{D\left(\stackrel{\circ}{W}_{1}, W_{2}, \varepsilon, \tau\right)\right\} .
\end{gathered}
$$

Из этого вычисления следует

$$
\mathbf{P}\left\{D_{q}\left(W_{1}, W_{2}, \varepsilon, \tau\right)\right\} \geqslant c_{26} \varepsilon \mathbf{P}\left\{D\left(\stackrel{\circ}{W}_{1}, W_{2}, \varepsilon, \tau\right)\right\} .
$$

С другой стороны, в силу самоподобия и предложения 2.1 , мы имеем

$$
\begin{aligned}
& \mathbf{P}\left\{D_{q}\left(W_{1}, W_{2}, \varepsilon, \tau\right)\right\}=\mathbf{P}\left\{D\left(W_{1}, W_{2}, \frac{\varepsilon}{\sqrt{q}}, \tau\right)\right\} \\
& \quad \leqslant c_{5} \exp \left(-\frac{\pi^{2} q^{2}}{8 \varepsilon^{2}}-\frac{c_{6} \tau^{1 / 3} q^{2}}{\varepsilon^{2}}\right) \leqslant c_{27} \exp \left(-\frac{\pi^{2}}{8 \varepsilon^{2}}-\frac{c_{6} \tau^{1 / 3}}{\varepsilon^{2}}\right) .
\end{aligned}
$$

С учетом (4.5), отсюда следует (4.3), т.е. мы доказали первую часть предложения.

Остается проверить вторую часть. Если $\tau^{1 / 3} \leqslant \varepsilon^{2}$, то доказывать нечего, так как, согласно (4.2), мы тривиальным образом имеем

$$
\begin{aligned}
& \mathbf{P}\left\{\left\|\stackrel{\circ}{W}_{1}\right\| \leqslant \varepsilon,\left\|\sqrt{1-\tau} \stackrel{\circ}{W}_{1}+\sqrt{\tau} \stackrel{\circ}{W}_{2}\right\| \leqslant \varepsilon\right\} \\
& \quad \leqslant \mathbf{P}\left\{\left\|\stackrel{\circ}{W}_{1}\right\| \leqslant \varepsilon\right\} \leqslant \frac{c_{28}}{\varepsilon} \exp \left\{-\frac{\pi^{2}}{8 \varepsilon^{2}}\right\} \leqslant \frac{c_{29}}{\varepsilon} \exp \left\{-\frac{\pi^{2}}{8 \varepsilon^{2}}-\frac{\tau^{1 / 3}}{\varepsilon^{2}}\right\} .
\end{aligned}
$$


Предположим теперь, что $\tau^{1 / 3}>\varepsilon^{2}$. Не ограничивая общности, при $i=1$ и 2 можем записать представления

$$
\stackrel{\circ}{W}_{i}(s):=W_{i}(s)-s W_{i}(1), \quad s \in[0,1] .
$$

Будем использовать запись $\stackrel{\circ}{W}_{i}=W_{i}-W_{i}(1) \mathrm{Id}$, где $W_{1}$ и $W_{2}-$ независимые броуновские движения. Отметим, что случайная величина $W_{2}(1)$ независима от процессов $\stackrel{\circ}{W}_{1}$ и $\stackrel{\circ}{W}_{2}$. Будем пользоваться неравенством $\mathbf{P}\left\{\left|W_{2}(1)\right| \leqslant x\right\} \geqslant x / 2$ при $x \in[0,1]$. Записывая

$$
p_{\varepsilon}:=\mathbf{P}\left\{\left\|\stackrel{\circ}{W}_{1}\right\| \leqslant \varepsilon,\left\|\sqrt{1-\tau} \stackrel{\circ}{W}_{1}+\sqrt{\tau} \stackrel{\circ}{W}_{2}\right\| \leqslant \varepsilon\right\},
$$

найдем

$$
\begin{gathered}
p_{\varepsilon}=\mathbf{P}\left\{\left\|\stackrel{\circ}{W}_{1}\right\| \leqslant \varepsilon,\left\|\sqrt{1-\tau} \stackrel{\circ}{W}_{1}+\sqrt{\tau} W_{2}-\sqrt{\tau} W_{2}(1) \operatorname{Id}\right\| \leqslant \varepsilon,\right. \\
\left.\left|W_{2}(1)\right| \leqslant \frac{\varepsilon^{3}}{\sqrt{\tau}}\right\}\left(\mathbf{P}\left\{\left|W_{2}(1)\right| \leqslant \frac{\varepsilon^{3}}{\sqrt{\tau}}\right\}\right)^{-1} \\
\leqslant \mathbf{P}\left\{\left\|\stackrel{\circ}{W}_{1}\right\| \leqslant \varepsilon,\left\|\sqrt{1-\tau} \stackrel{\circ}{W}_{1}+\sqrt{\tau} W_{2}\right\| \leqslant \varepsilon+\varepsilon^{3}\right\}\left(\frac{\varepsilon^{3}}{2 \sqrt{\tau}}\right)^{-1} .
\end{gathered}
$$

В свете соотношения (4.3), которое мы уже доказали, получается

$$
\begin{aligned}
p_{\varepsilon} & \leqslant 2 c_{21} \frac{\sqrt{\tau}}{\varepsilon^{4}} \exp \left(-\frac{\pi^{2}}{8\left(\varepsilon+\varepsilon^{3}\right)^{2}}-\frac{c_{22} \tau^{1 / 3}}{\left(\varepsilon+\varepsilon^{3}\right)^{2}}\right) \\
& \leqslant c_{30} \frac{\sqrt{\tau}}{\varepsilon^{4}} \exp \left(-\frac{\pi^{2}}{8 \varepsilon^{2}}-\frac{c_{22} \tau^{1 / 3}}{\varepsilon^{2}}\right) .
\end{aligned}
$$

Отсюда вытекает (4.4), и доказательство предложения 4.1 завершено.

5. Замечания и вопросы. Этот заключительный раздел содержит ряд дополнительных замечаний и вопросов, связанных с нижними функциями бесконечномерных броуновских движений и эмпирических процессов.

1. Стандартная процедура обращения времени броуновского движения мгновенно приводит к следующему аналогу теоремы 1.1 для малых времен. Пусть поле $X$ задано формулой (1.1). Если $f>0$ не убывает, то

$$
\begin{aligned}
& \int^{\infty} \frac{f^{6}(t)}{t} \exp \left(-\frac{\pi^{2}}{8} f^{2}(t)\right) d t\left\{\begin{array}{l}
<\infty \\
=\infty
\end{array}\right. \\
& \Longleftrightarrow \mathbf{P}\left\{\|X(t)\| \leqslant \frac{t^{1 / 2}}{f(1 / t)} \text { б.ч. при } t \rightarrow 0\right)=\left\{\begin{array}{l}
0 \\
1
\end{array},\right.
\end{aligned}
$$

где норма $\|\cdot\|$ определена в (1.2).

2. Наша статья посвящена изучению скорости ухода на бесконечность конкретного бесконечномерного броуновского движения в конкретном пространстве (рассматривается броуновский лист и равномерная 
норма). Было бы очень интересно охарактеризовать скорости ухода для более общих бесконечномерных броуновских движений. Сравнивая две части (2.1) и (2.2) теоремы 1.1, отметим, что (2.1) без особых усилий можно распространить на любое бесконечномерное броуновское движение $X$ со значениями в сепарабельном банаховом пространстве общего вида $(E,\|\cdot\|)$, если только имеется достаточно хорошая информация об асимптотическом поведении $\mathbf{P}\{\|X(1)\| \leqslant \varepsilon\}$. Напротив, часть (2.2) представляется гораздо более специфичной - именно здесь нам пришлось использовать специальные свойства нашего примера. Мы также задаемся следуюшим вопросом: правда ли, что вероятность $\mathbf{P}\{\|X(1)\| \leqslant \varepsilon\}$ полностью определяет скорость ухода $X$ на бесконечность?

3. Если быть менее амбициозными, можно рассмотреть все тот же броуновский лист $\mathbf{M}$ относительно $L^{2}$-нормы $\|g\|_{2}:=\left[\int_{0}^{1} g^{2}(t) d t\right]^{1 / 2}$. Возможно ли охарактеризовать $\mathbf{P}\left\{\|\mathbf{M}(\cdot, t)\|_{2} \leqslant \sqrt{t} / f(t)\right.$ б.ч. $\}$ с помощью некоторого интегрального критерия? Частичный ответ находится в работе Альбина [5]. Используя его теорему 4, получаем, что для любого $T>1$ верно

$$
\mathbf{P}\left\{\min _{1 \leqslant t \leqslant T} \frac{\|\mathbf{M}(\cdot, t)\|_{2}}{\sqrt{t}} \leqslant \varepsilon\right\} \sim \frac{c \ln T}{\varepsilon^{3}} \exp \left\{-\frac{1}{8 \varepsilon^{2}}\right\}, \quad \varepsilon \rightarrow 0 .
$$

По лемме Бореля-Кантелли

$$
\int^{\infty} \frac{f^{3}(t)}{t} \exp \left(-\frac{f^{2}(t)}{8}\right) d t<\infty \Longrightarrow \mathbf{P}\left\{\|\mathbf{M}(\cdot, t)\|_{2} \leqslant \frac{t^{1 / 2}}{f(t)} \text { б.ч. }\right\}=0 .
$$

Аналогично и для процесса Кифера имеем

$$
\int^{\infty} \frac{f^{4}(t)}{t} \exp \left(-\frac{f^{2}(t)}{8}\right) d t<\infty \Longrightarrow \mathbf{P}\left\{\left\|K_{n}\right\|_{2} \leqslant \frac{n^{1 / 2}}{f(n)} \text { б.ч. }\right\}=0 .
$$

Оба интеграла в левых частях наводят на мысль, что здесь критической подпоследовательностью должна быть $t_{i}:=\exp \left(i /(\ln i)^{2}\right)$. K сожалению, насколько нам известно, проблема поиска необходимого и достаточного теста пока остается открытой.

4. Подпоследовательность Эрдёша не впервые оказывается непригодной для исследования тонкого интегрального теста. Так, Альбин [4], занимавшийся характеризацией верхних функций интеграла квадрата одномерного броуновского движения, показал, что подпоследовательность Эрдёша не подходит для решения данной задачи.

Часть этой работы была выполнена во время визита первого автора в университет Париж VI в апреле 2001 г. Мы благодарны за оказанное гостеприимство Лаборатории теории вероятностей и стохастических моделей (UMR 7599). 


\section{СПИСОК ЛИТЕРАТУРЫ}

1. Ито К., Маккин Г. П. Диффузионные процессы и их траектории. М.: Мир, 1968, $394 \mathrm{c}$.

2. Лифшии М. А. Гауссовские случайные функции. Киев: ТВиМС, 1995, 256 с.

3. Могульский $A$. A. О законе повторного логарифма в форме Чжуна для функциональных пространств. - Теория вероятн. и ее примен., 1979, т. 24 , в. 2, с. 399407.

4. Albin J. M. P. Upper and lower classes for $L^{2}$ - and $L^{p}$-norms of Brownian motion and norms of $\alpha$-stable motion. - Stochastic Process. Appl., 1995, v. 58, № 1, p. 91-103.

5. Albin J. M. P. Minima of $H$-valued Gaussian processes. - Ann. Probab., 1996, v. 24, № 2 , p. $788-824$.

6. Breiman $L$. A delicate law of the iterated logarithm for non-decreasing stable processes. - Ann. Math. Statist., 1968, v. 39, p. 1818-1824; 1969, v. 40, p. 1855.

7. Chung K.L. On the maximum partial sums of sequence of independent random variables. - Trans. Amer. Math. Soc., 1948, v. 64, p. 205-233.

8. Cox D.D. On the existence of natural rate of escape functions for infinite dimensional Brownian motions. - Ann. Probab., 1982, v. 10, p. 623-638.

9. Csáki E. An integral test for the supremum of Wiener local time. -- Probab. Theory Related Fields, 1989, v. 83, № 1, p. 207-217.

10. Dvoretzky A., Erdös P. Some problems on random walk in space. - Proceedings of the Second Berkeley Symposium on Mathematical Statistics and Probability. Berkeley: Univ. of California Press, 1951, p. 353-367.

11. Erdös P. On the law of the iterated logarithm. -- Ann. Math., 1942, v. 43, p. 419-436.

12. Erickson K.B. Rates of escape of infinite dimensional Brownian motion. - Ann. Probab., 1980 , v. 8, p. 325-338.

13. Khoshnevisan D. Multiparameter Processes: An Introduction to Random Fields. New York: Springer-Verlag, 2002.

14. Kochen S. B., Stone C. J. A note on the Borel-Cantelli lemma. - Illinois J. Math., 1964 , v. 8 , p. $248-251$.

15. Kolmogorov A.N. Sulla determinazione empirica di una legge di distribuzione. Giorn. Ist. Ital. Attuari, 1933, v. 4, p. 83-91; рус. пер.: Колмогоров А.Н. Об эмпирическом определении закона распределения. - Теория вероятностей и математическая статистика. М.: Наука, 1986, с. 134-141.

16. Komlós J., Major P., Tusnády G. An approximation of partial sums of independent RV's and the sample DF. II. - Z. Wahrscheinlichkeitstheor. verw. Geb., 1976, v. 34, p. 33-58.

17. Kuelbs J. Rates of growth for Banach space valued independent increment processes. - Lecture Notes in Math., 1979, v. 709, p. 151-169.

18. Shorack G. R., Wellner J. A. Empirical Processes with Applications to Statistics. New York: Wiley, 1986, $938 \mathrm{p}$. 